\title{
Detailed study of the $K_{e 4}$ decay mode properties
}

\author{
Milena Misheva*i \\ Joint Institute for Nuclear Research, Dubna, Russia \\ E-mail: dyulendarova.milena@gmail.com
}

The largest data samples of semi-leptonic charged kaon decays in $K_{e 4}^{+-}\left(K^{ \pm} \rightarrow \pi^{+} \pi^{-} e^{ \pm} v_{e}\right)$ and $K_{e 4}^{00}\left(K^{ \pm} \rightarrow \pi^{0} \pi^{0} e^{ \pm} v_{e}\right)$ modes have been collected by the NA48/2 experiment at CERN SPS. We report improved measurements of branching fractions and detailed form factors studies. The achieved remarkable precisions of the results bring new inputs to low energy QCD description and crucial tests of predictions from Chiral Perturbation Theory.

XXII. International Workshop on Deep-Inelastic Scattering and Related Subjects, 28 April - 2 May 2014

Warsaw, Poland

\footnotetext{
* Speaker.

$\dagger$ on behalf of the NA48/2 Collaboration: G. Anzivino, R. Arcidiacono, S. Balev, J.R. Batley, M. Behler, S. Bifani, C. Biino, A. Bizzeti, B. Bloch-Devaux, G. Bocquet, N. Cabibbo, M. Calvetti, N. Cartiglia, A. Ceccucci, P. Cenci, C. Cerri, C. Cheshkov, J.B. Chèze, M. Clemencic, G. Collazuol, F. Costantini, A. Cotta Ramusino, D. Coward, D. Cundy, A. Dabrowski, P. Dalpiaz, C. Damiani, M. De Beer, J. Derré, H. Dibon, L. DiLella, N. Doble, K. Eppard, V. Falaleev, R. Fantechi, M. Fidecaro, L. Fiorini, M. Fiorini, T. Fonseca Martin, P.L. Frabetti, L. Gatignon, E. Gersabeck, A. Gianoli, S. Giudici, A. Gonidec, E. Goudzovski, S. Goy Lopez, M. Holder, P. Hristov, E. Iacopini, E. Imbergamo, M. Jeitler, G. Kalmus, V. Kekelidze, K. Kleinknecht, V. Kozhuharov, W. Kubischta, G. Lamanna, C. Lazzeroni, M. Lenti, L. Litov, D. Madigozhin, A. Maier, I. Mannelli, F. Marchetto, G. Marel, M. Markytan, P. Marouelli, M. Martini, L. Masetti, E. Mazzucato, A. Michetti, I. Mikulec, N. Molokanova, E. Monnier, U. Moosbrugger, C. Morales Morales, D.J. Munday, A. Nappi, G. Neuhofer, A. Norton, M. Patel, M. Pepe, A. Peters, F. Petrucci, M.C. Petrucci, B. Peyaud, M. Piccini, G. Pierazzini, I. Polenkevich, Yu. Potrebenikov, M. Raggi, B. Renk, P. Rubin, G. Ruggiero, M. Savrié, M. Scarpa, M. Shieh, M.W. Slater, M. Sozzi, S. Stoynev, E. Swallow, M. Szleper, M. Valdata-Nappi, B. Vallage, M. Velasco, M. Veltri, S. Venditti, M. Wache, H. Wahl, A. Walker, R. Wanke, L. Widhalm, A. Winhart, R. Winston, M.D. Wood, S.A. Wotton, O. Yushchenko, A. Zinchenko, M. Ziolkowski.
} 


\section{Introduction}

Kaon decays are perfect for studying low energy strong interactions. Semi-leptonic four-body decays are of particular interest because of the small number of hadrons in the final state which are related to the investigation of $\pi \pi$ interaction. The global analysis of $\pi \pi, \pi K$ and $K_{e 4}$ data allow the determination of Low Energy Constants of Chiral Perturbation Theory (ChPT) at Leading and Next to Leading Orders $[1,2]$ and subsequent predictions of form factors and branching fractions.

A brief introduction to the NA48/2 experiment, concise descriptions of the event selections and obtained measurements of $K_{e 4}$ branching ratios and form factors are reported.

\section{The NA48/2 experiment}

The beam line of the NA48/2 experiment was designed to produce two simultaneous $K^{+}$and $K^{-}$beams by $400 \mathrm{GeV} / \mathrm{c}$ primary protons delivered by the CERN SPS and impinging on a $400 \mathrm{~mm}$ long beryllium target. The opposite charged particles are selected with a momentum range of (60 \pm 3 ) $\mathrm{GeV} / \mathrm{c}$ by two systems of dipole magnets with zero total deflection, focusing quadrupoles, muon sweepers and collimators. The positive and negative kaon beams travel further through a 114 $\mathrm{m}$ vacuum decay volume. Their axes coincide within $1 \mathrm{~mm}$. A detailed description of the NA48 experimental set-up can be found in [3]. The momenta of the charged decay products are measured by a magnetic spectrometer located in a tank filled with helium at nearly atmospheric pressure. It consists of four octagonal drift chambers $(\mathrm{DCH})$ and a large aperture dipole magnet. The momentum resolution achieved in the spectrometer is $\sigma(p) / p=(1.02 \oplus 0.044 p) \%(\mathrm{p}$ in GeV/c). A fast signal for the trigger is provided by a scintillator hodoscope (HOD) situated just after the spectrometer. It measures the time of arrival of charged particles with an offline resolution of $\sim 150$ ps. Downstream of the HOD a quasi-homogeneous liquid Krypton calorimeter ( $\mathrm{LKr}$ ) is placed. It is used to measure the energy deposits of photons and electrons. The calorimeter is $27 X_{0}$ deep and its energy resolution is $\sigma(E) / E=(3.2 / \sqrt{E} \oplus 9.0 / E \oplus 0.42) \%$ ( $\mathrm{E}$ in $\mathrm{GeV})$. The muon veto counter (MUV) is the last component of the NA48/2 detector set-up. It is composed of three planes of alternating horizontal and vertical scintillator strips separated by $80 \mathrm{~cm}$ thick iron walls. Its time resolution is below $1 \mathrm{~ns}$ and about a per mil inefficiency for muons with momenta higher than 10 $\mathrm{GeV} / \mathrm{c}$.

\section{3. $K_{e 4}$ Braching ratio measurements}

The branching ratio (BR) of each $K_{e 4}$ mode is measured relative to a normalization decay using the following formula:

$$
B R\left(K_{e 4}^{+-}\right)=\frac{N_{s}-N_{b}}{N_{n}} \cdot \frac{A_{n} \varepsilon_{n}}{A_{s} \varepsilon_{s}} \cdot B R(n)
$$

where $N_{s}, N_{b}, N_{n}$ are the numbers of signal, background and normalization events, $A_{s}$ and $\varepsilon_{s}\left(A_{n}\right.$ and $\varepsilon_{n}$ ) are the geometrical acceptance and trigger efficiency for the signal (normalization) sample, respectively. The normalization branching ratio $B R(n)$ is taken from world average [4]. 
NA48/2 has measured the BR of the charged and neutral $K_{e 4}$ modes by using for reference channels $K^{ \pm} \rightarrow \pi^{+} \pi^{-} \pi^{ \pm}\left(K_{3 \pi}^{+-}\right)$and $K^{ \pm} \rightarrow \pi^{0} \pi^{0} \pi^{ \pm}\left(K_{3 \pi}^{00}\right)$, respectively. The signal and the normalization samples are collected concurrently using the same trigger logic and a common event selection is considered as much as possible in order to cancel partially systematic effects.

Detailed GEANT-3-based Monte Carlo simulations are used to compute the acceptances of the signal and normalization channels in both analyses. Each simulation includes full detector and material description, DCH alignment, DCH and LKr local inefficiencies and kaon beam properties throughout the running period.

\section{1 $K^{ \pm} \rightarrow \pi^{ \pm} \pi^{0} e^{ \pm} v_{e}$ decay}

The $K_{e 4}^{+-}$and $K_{3 \pi}^{+-}$candidates are reconstructed from three charged tracks which form one common vertex in the decay volume and satisfy all requirements of DCH, HOD, LKr and MUV geometrical acceptances. The ratio of energy deposition in the LKr calorimeter and the momentum measured by the spectrometer $(\mathrm{E} / \mathrm{p})$ is used for pion/electron identification. The track is identified as an electron if its momentum is greater than $2.75 \mathrm{GeV} / \mathrm{c}$ and it has an associated cluster with $0.9<E / p<1.1$. The criteria for pion identification require momentum larger than $5 \mathrm{GeV} / \mathrm{c}$ and E/p smaller than 0.8. The electric charge of the two pions should be 0. Additional suppression of pions mis-identified as electrons is achieved by using a dedicated discriminant variable defined as a linear combination of quantities related to shower properties in the electromagnetic calorimeter (such as E/p, radial shower width and energy weighted track-cluster distance at the LKr front face). Rejection of the significant $K_{3 \pi}^{+-}$background source is obtained by requiring the invariant mass $\left(M_{3 \pi}\right)$ of the three tracks in $\pi^{+} \pi^{-} \pi^{ \pm}$hypothesis and the transverse momentum relative to the beam axis $\left(p_{t}\right)$ to be outside of an half-ellipse centred on the PDG value of the kaon mass [4] and zero $p_{t}$, with semi-axes $20 \mathrm{MeV} / \mathrm{c}^{2}$ and $35 \mathrm{MeV} / \mathrm{c}$, respectively. The squared missing mass is required to be larger than $0.04(\mathrm{GeV} / \mathrm{c})^{2}$ in order to remove $K^{ \pm} \rightarrow \pi^{ \pm} \pi^{0}$ events with a subsequent $\pi^{0} \rightarrow e^{+} e^{-} \gamma$ decay. The normalization $K_{3 \pi}^{+-}$sample is selected by applying all possible common criteria with the main channel and additionally demanding the $M_{3 \pi}$ and the $p_{t}$ to be inside a smaller ellipse with semi-axes $12 \mathrm{MeV} / \mathrm{c}^{2}$ and $25 \mathrm{MeV} / \mathrm{c}$ respectively.

A total sample of $1.1 \times 10^{6} K_{e 4}^{+-}$candidate-events $\left(712288 \mathrm{~K}^{+}\right.$and $\left.396653 \mathrm{~K}^{-}\right)$and prescaled $18.8 \times 10^{6} K_{3 \pi}$ candidates $\left(12.09 \times 10^{6} K^{+}\right.$and $\left.6.73 \times 10^{6} K^{-}\right)$are selected from data recorded during 2003-2004.

The main remaining background is the $K^{ \pm} \rightarrow \pi^{+} \pi^{-} \pi^{ \pm}$channel followed by the decay in flight of a single pion $\pi^{ \pm} \rightarrow e^{ \pm} v$ or mis-identification of a pion as an electron. Another background source is $K^{ \pm} \rightarrow \pi^{0}\left(\pi^{0}\right) \pi^{ \pm}$with subsequent Dalitz decay of $\pi^{0} \rightarrow e^{+} e^{-} \gamma$, where the $\pi^{ \pm}$fakes the electron response and photon(s) is (are) undetected. The total background contamination in the signal region is of order $1 \%$.

The final result is a weighted average of 16 values obtained in eight independent data subsamples and both kaon charges. The obtained $K_{e 4}^{+-}$branching ratio by NA48/2 [5] is $B R\left(K_{e 4}^{+-}\right)=$ $\left(4.257 \pm 0.004_{\text {stat. }} \pm 0.016_{\text {syst. }} \pm 0.031_{\text {ext. }}\right) \times 10^{-5}$. The precision of the result is 3 times better than the available PDG value [4].

$3.2 K^{ \pm} \rightarrow \pi^{0} \pi^{0} e^{ \pm} v_{e}$ decay

The neutral $K_{e 4}^{00}$ and $K_{3 \pi}^{00}$ candidates are selected by requiring one charged track and at least four 
photons, forming two $\pi^{0}$ 's, pointing to the same vertex. The track is reconstructed by the magnetic spectrometer and the photons are identified as electromagnetic showers, developed in the $\mathrm{LKr}$, without associated track and with energy above $3 \mathrm{GeV}$. For each track candidate with momentum larger than $5 \mathrm{GeV} / \mathrm{c}$, the charged vertex longitudinal position $\left(Z_{c}\right)$ is defined as the closest distance of approach to the beam line smaller than $5 \mathrm{~cm}$. On the other hand, the reconstructed longitudinal positions of both $\pi^{0} \rightarrow \gamma \gamma$ decay candidates $\left(Z_{1}\right.$ and $\left.Z_{2}\right)$ are required to be within $500 \mathrm{~cm}$. The neutral vertex, $Z_{n}=\left(Z_{1}+Z_{2}\right) / 2$, is determined as the average longitudinal positions of both $\pi^{0}$ s. Track candidate for which $Z_{c}$ and $Z_{n}$ are compatible within $\pm 800 \mathrm{~cm}$ is retained further in the analysis.

The track is identified as an electron if it has an associated cluster with E/p between 0.9 and 1.1 or as a charged pion if its momentum is above $5 \mathrm{GeV} / \mathrm{c}$ (it may have an associated cluster or not). Powerful suppression of pions mis-identified as electrons is obtained by using the above mentioned discriminant variable as in $K_{e 4}^{ \pm}$decay analysis.

The separation between the signal candidates from the abundant $K_{3 \pi}^{00}$ decays is obtained by means of an elliptic requirement in the plane of missing mass $\left(M_{3 \pi}-M_{K}\right)$ and missing transverse momentum $\left(p_{t}\right)$ assuming the $\pi^{0} \pi^{0} \pi^{ \pm}$decay hypothesis. $M_{3 \pi}$ is the reconstructed three pion invariant mass and $M_{K}$ is the nominal kaon mass [4]. $K_{e 4}^{00}$ events are selected outside an ellipse centered on the nominal $K^{ \pm}$mass and $5 \mathrm{MeV} / \mathrm{c} p_{t}$, with semi-axes of $15 \mathrm{MeV} / \mathrm{c}^{2}$ and $30 \mathrm{MeV} / \mathrm{c}$, respectively. $K_{3 \pi}^{00}$ candidates are required to be inside an ellipse with the same center but with semi-axes of $10 \mathrm{MeV} / \mathrm{c}^{2}$ and $20 \mathrm{MeV} / \mathrm{c}$ respectively.

The invariant mass of the electron-neutrino system is required to be between 0 and 0.25 $\mathrm{GeV} / c^{2}$.

A total sample of $65210 K_{e 4}^{00}$ events and almost 94 million $K_{3 \pi}^{00}$ are selected and used for the branching ratio measurement.

The background from fake-electron tracks is evaluated to be $0.65 \%$ of the signal events and a contribution of $0.12 \%$ comes from the $K_{3 \pi}^{00}$ channel with subsequent $\pi^{ \pm} \rightarrow e^{ \pm} v$ decay. The background of accidental coincidence of another kaon decay with an additional track or photon and resulting in the same final state topology as the $K_{e 4}^{00}$ signal is estimated as $0.23 \%$. The total background to signal contamination is $1 \%$.

The value of the branching ratio is measured by NA48/2 [6] as the combination of ten statistically independent samples: $B R\left(K_{e 4}^{00}\right)=\left(2.552 \pm 0.010_{\text {stat. }} \pm 0.010_{\text {syst. }} \pm 0.032_{\text {ext. }}\right) \times 10^{-5}$, where the error is dominated by the external uncertainty from the branching fraction of the normalization decay mode. It is 13 times more precise measurement than the PDG corresponding value [4]. The systematic uncertainty includes contributions from the control of background estimate, radiative effects, trigger efficiency and acceptance.

\section{Form factor measurement}

The decay amplitude of the $K_{e 4}^{+-}$is a product of the weak leptonic and (V-A) hadronic currents. The latter is described in terms of three axial-vector $(F, G, R)$ and one vector $(H)$ complex form factors. The $\mathrm{R}$ form factor is not taken into account in the final expression of the $K_{e 4}$ matrix element because it is always multiplied by the squared electron mass and therefore can be neglected. The remaining form factors may be developed in a partial wave expansion with respect to 
$\cos \theta_{\pi}$. We limit the expansions to $\mathrm{S}$ - and P-waves and consider a unique phase $\left(\delta_{p}\right)$ for all P-waves contributions (in absence of CP-violating weak phases):

$$
F=F_{s} e^{i \delta_{f s}}+F_{p} e^{i \delta_{f p}} \cos \theta_{\pi}, \quad G=G_{p} e^{i \delta_{g p}} \cos \theta_{\pi}, \quad H=H_{p} e^{i \delta_{h p}} \cos \theta_{\pi}
$$

Therefore the decay amplitude depends on the real form factor magnitudes $F_{s}, F_{p}, G_{p}, H_{p}$, a single phase shift $\delta=\delta_{s}-\delta_{p}$ and five Cabibbo-Maksymowicz variables [7] which fully describe the $K_{e 4}$ kinematics (the squared invariant masses of dipion $\left(S_{\pi}\right)$ and dilepton $\left(S_{e}\right)$ systems, the angle $\theta_{\pi}\left(\theta_{e}\right)$ of the $\pi^{ \pm}\left(e^{ \pm}\right)$in the dipion (dilepton) rest frame with respect to the the flight direction of dipion (dilepton) in the $K^{ \pm}$center-of-mass system, and the angle $\phi$ between dipion and dilepton planes).

The $K_{e 4}$ decay form factors can be expressed as a series expansion of dimensionless invariants $q^{2}=\left[\left(S_{\pi} / 4 m_{\pi}^{2}\right)-1\right]$ and $\left(S_{e} / 4 m_{\pi}^{2}\right)$. All form factor values are obtained relative to a common value $f_{s}$ which represents the $\mathrm{S}$-wave axial vector form factor, $F_{s}$, at $q^{2}=0$ and $S_{e}=0$ :

$$
\begin{aligned}
& F_{s} / f_{s}=1+\left(f_{s}^{\prime} / f_{s}\right) q^{2}+\left(f_{s}^{\prime \prime} / f_{s}\right) q^{4}+\left(f_{e}^{\prime} / f_{s}\right) S_{e} / 4 m_{\pi}^{2} \\
& F_{p} / f_{s}=f_{p} / f_{s}, \quad G_{p} / f_{s}=g_{p} / f_{s}+\left(g_{p}^{\prime} / f_{s}\right) q^{2}, \quad H_{p} / f_{s}=h_{p} / f_{s} .
\end{aligned}
$$

The $K_{e 4}^{+-}$hadronic form factors and the S-wave $\pi \pi$ scattering lengths with $I=0$ and $I=2, a_{0}^{0}$ and $a_{2}^{0}$, have been extensively studied by NA48/2 and all results have been published in [8].

In the neutral $K_{e 4}^{00}$ mode, the presence of two identical particles in the final state, reduces the independent kinematic variables to three: $S_{\pi}, S_{e}$ and $\theta_{e}$. Moreover, only one form factor $\left(F_{s}\right)$ enters the decay amplitude expression because the dipion system can have only isospin $I=0$ and orbital momentum $l=0$. The allowed kinematic range of $q^{2}$ spans both positive and negative values. The ratio of data and simulation (including a constant form factor) distributions projected on the $q^{2}$ axis is shown on Fig.1. A quadratic behaviour is observed for the positive $q^{2}$ values with the same parameterization (4.1) of the $F_{s} / f_{s}$ as in the analysis of $K_{e 4}^{ \pm}$, and a deficit of events is observed below the $2 m_{\pi^{+}}$threshold $\left(q^{2}=0\right)$. The following fitting function $F_{s} / f_{s}=1+d \sqrt{\left|q^{2} /\left(1+q^{2}\right)\right|}+$ $\left(f_{e}^{\prime} / f_{s}\right) S_{e} / 4 m_{\pi}^{2}$ is taken for $q^{2}<0$. This cusp-like shape at the $2 m_{\pi}^{+}$threshold can be explained by the final state charge exchange scattering $\left(\pi^{+} \pi^{-} \rightarrow \pi^{0} \pi^{0}\right)$ as in the $K_{3 \pi}^{00}$ mode [9].

The results are in agreement with NA48/2 $K_{e 4}^{+-}$analysis: $f_{s}^{\prime} / f_{s}=0.149 \pm 0.033_{\text {stat }} \pm 0.014_{\text {syst }}$, $f_{s}^{\prime \prime} / f_{s}=-0.070 \pm 0.039_{\text {stat }} \pm 0.013_{\text {syst }}, f_{e}^{\prime} / f_{s}=0.113 \pm 0.022_{\text {stat }} \pm 0.07_{\text {syst }}, d=-0.256 \pm 0.049_{\text {stat }} \pm$ $0.016_{\text {syst }}$. More details can be found in [6].

\section{Summary}

More than one million $K_{e 4}^{+-}$decay candidates with less than one percent background has been collected by the NA48/2 experiment at CERN SPS in 2003-2004. The rich statistics gives a great opportunity to perform a detailed study of decay properties. The BR $\left(K_{e 4}^{+-}\right)$, inclusive of $K_{e 4 \gamma}^{+-}$mode, is obtained with total relative error of $0.8 \%$. This measurement complements the investigation of $\mathrm{S}$ - and P-wave hadronic form factors [5].

The $K_{e 4}^{00}$ Branching ratio, inclusive of radiative decays, has been measured by NA48/2 from a sample of 65210 events with $1 \%$ background contamination. The first measurement of the hadronic form factor has been obtained including its variation in the $\left(S_{\pi}, S_{e}\right)$-plane and providing an evidence for final state charge exchange scattering $\left(K_{e 4}^{+-}\right)$in the decay mode below the $2 m_{\pi^{+}}$threshold [6]. 


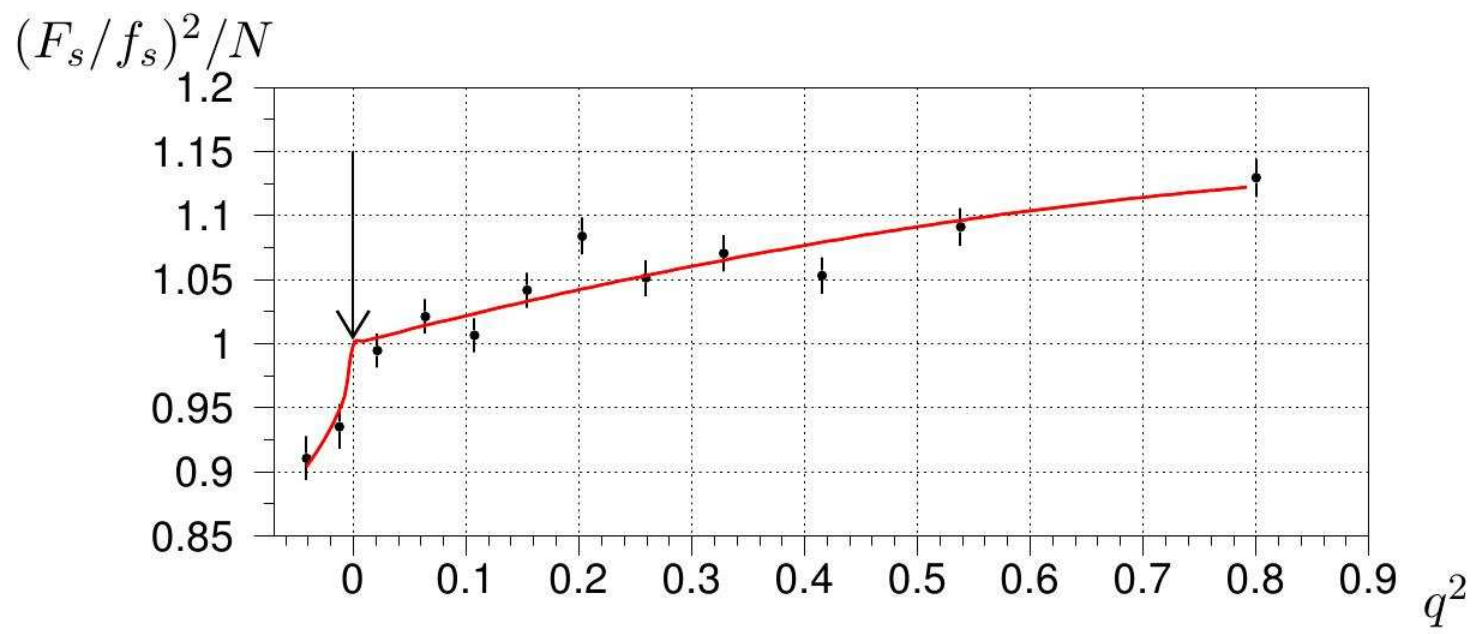

Figure 1: Data and simulation (including a constant form factor) $q^{2}$ distributions in equal population bins. Each symbol is plotted at the barycenter position of the data events in the bin. The line gives the empirical description using the best fit-parameter values: a 2-degree polynomial above $q^{2}=0$ and a cusp-like function below. $\mathrm{N}$ is a normalization factor.

The analysis of the $K^{ \pm} \rightarrow \pi^{0} \pi^{0} \pi^{ \pm}$performed by NA48/2 has enlightened a cusp in the $M_{\pi^{0}} \pi^{0}$ distribution which may be explained by re-scattering effects in the $\pi \pi$ system around the $2 m_{\pi^{+}}$ threshold [9]. Neglecting potential (but small) common systematic contributions to the experimental errors, the results from both $K_{3 \pi}$ and $K_{e 4}^{+-}$analyses have been combined in more precise scattering lengths measurements:

$$
\begin{aligned}
a_{0}^{0} & =0.2210 \pm 0.0047_{\text {stat }} \pm 0.0040_{\text {syst }}, \quad a_{0}^{0}(\mathrm{ChPT})=0.220 \pm 0.005_{t h} \\
a_{0}^{2} & =-0.0429 \pm 0.0044_{\text {stat }} \pm 0.0028_{\text {syst }}, \quad a_{0}^{2}(\mathrm{ChPT})=-0.0444 \pm 0.0010_{t h} \\
a_{0}^{0}-a_{0}^{2} & =0.2639 \pm 0.0020_{\text {stat }} \pm 0.0015_{\text {syst }}, \quad a_{0}^{0}-a_{0}^{2}(\mathrm{ChPT})=0.264 \pm 0.004_{t h}
\end{aligned}
$$

where the corresponding values of scattering lengths calculated by ChPT are quoted for comparison. These results provide a stringent test of ChPT predictions.

\section{References}

[1] J. Bijnens, G. Colangelo, J. Gasser, Nucl.Phys.B427 (1994) 427

[2] J.Bijnens and I. Jemos, Nucl.Phys B854 (2012) 631

[3] NA48: V. Fanti et al., Nucl. Instrum. Meth. A574 (2007) 433

[4] PDG: J. Beringer et al., Phys. Rev. D86 (2012) 010001

[5] NA48/2: Batley, J.R. et al., Phys. Lett. B715 (2012) 105

[6] NA48/2:Batley, J.R. et al., CERN-PH-EP-2014-145, [arXiv:1406.4749] (2014)

[7] N. Cabibbo and A. Maksymowicz, Phys. Rev. 137 (1965) B438; Phys. Rev. 168 (1968) 1926

[8] NA48/2: Batley, J.R. et al., Eur.Phys.J. C70 (2010) 635

[9] NA48/2: Batley, J.R. et al., Eur.Phys.J. C64, (2009) 589 